\title{
Fecal Impaction Dystocia
}

\author{
${ }^{1}$ Mayoor M Daigavane, ${ }^{2}$ Parmanand K Shah, ${ }^{3}$ Hemangi Choudhari
}

\begin{abstract}
Fecoliths cause many complications in pregnancy, such as dystocia, rupture uterus, and malpresentation. Obstructed labor due to a fecolith in the rectum and colon is very rare. We are presenting a case of obstructed labor and malpresentation caused by a large fecolith.
\end{abstract}

Keywords: Dystocia, Fecoliths, Malpresentation, Obstructed labor, Rupture uterus.

How to cite this article: Daigavane MM, Shah PK, Choudhari $\mathrm{H}$. Fecal Impaction Dystocia. J South Asian Feder Obst Gynae 2018;10(1):66-68.

\section{Source of support: Nil}

Conflict of interest: None

Date of received: 15 January 2018

Date of acceptance: 25 February 2018

Date of publication: May 2018

\section{INTRODUCTION}

Obstetric literature contains references to various seldom encountered conditions-including abnormalities of the generative tract and contiguous structureswhich contribute to "soft tissue dystocia." One such adjacent structure, the rectum, has been implicated as a cause of dystocia in many conditions, one of them being fecal impaction dystocia. Fecal impaction as a cause of obstructed labor is a very rare situation with a countable number of cases having been reported worldwide.

\section{CASE REPORT}

A 23-year-old G5 P4 L1 FSB2 intrauterine fetal death 1 (IUFD1), with previous one lower segment cesarean section (LSCS) and previous hysterotomy with manual removal of feces with suprapubic cystolithotomy done in

\footnotetext{
${ }^{1}$ Assistant Professor, ${ }^{2}$ Professor and Head, ${ }^{3}$ Associate Professor

${ }^{1-3}$ Department of Obstetrics and Gynecology, Seth Gordhandas Sunderdas Medical College and King Edward Memorial Hospital Mumbai, Maharashtra, India

Corresponding Author: Mayoor M Daigavane, Assistant Professor, Department of Obstetrics and Gynecology, Seth Gordhandas Sunderdas Medical College and King Edward Memorial Hospital, Mumbai, Maharashtra, India, Phone: +917709160220, e-mail: dr.anirbandasgupta@gmail.com
}

view of obstructed labor with impending uterine rupture due to fecal impaction and bladder stone, registered antenatally with us at 16 weeks of gestation. Patient was a case of chronic constipation with poor compliance to stool softeners. The cause of chronic constipation was not known. The patient had no family history and the chronic constipation could be most likely due to poor dietary and bowel habits. She did not have complete bowel evacuation for 2 months. She followed up at 30 weeks gestation with chief complaints of constipation. On per vaginal examination, cervix could not be palpated due to hard mass in the rectum lifting up the posterior vaginal wall. She was admitted and manual removal of fecal matter was done per rectally. On day 3 of admission, she went in spontaneous labor and antepartum hemorrhage. Fetal foot could be palpated per vaginally. Emergency LSCS was done and intraoperatively scar dehiscence was observed with bladder advanced on the lower uterine segment with dense adhesions (Fig. 1). Baby was found in breech presentation, hyperflexed, stillbirth, and placenta was anterior, separated from the myometrium (Fig. 2). After cesarean section, abdomen was explored and the bowel was found to be distended up to the splenic flexure of the colon. Sigmoid colon was $17.5 \mathrm{~cm}$ dilated over a length of $22.5 \mathrm{~cm}$ and filled with hard stools (Fig. 3). Surgeons performed manual fecal removal of the impacted feces per rectally with simultaneous milking of the megacolon per abdomen. Bowel was evacuated completely and the patient was started on stool softeners post cesarean. She passed soft stools on day 3 and regularly thereafter. She was advised high-fiber diet with plenty of oral fluids and regular follow-up with surgery clinic.

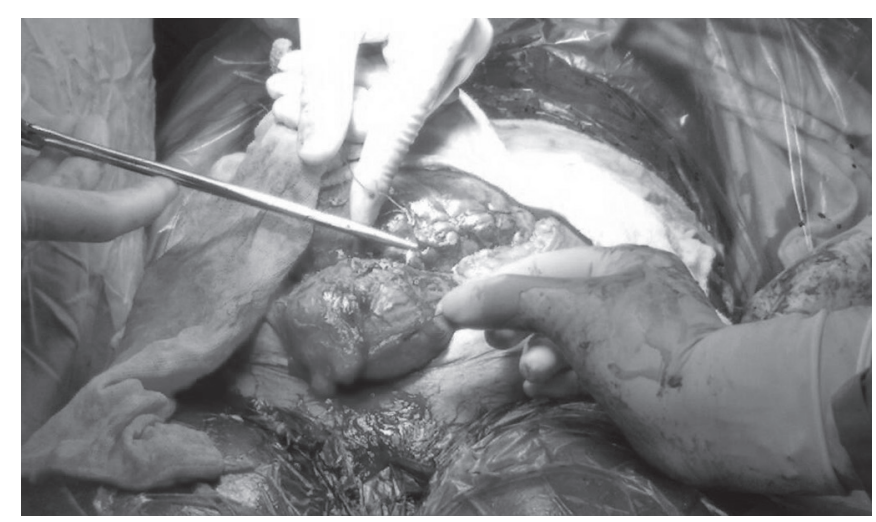

Fig. 1: Bladder adherent to lower uterine segment. Separated by sharp dissection 


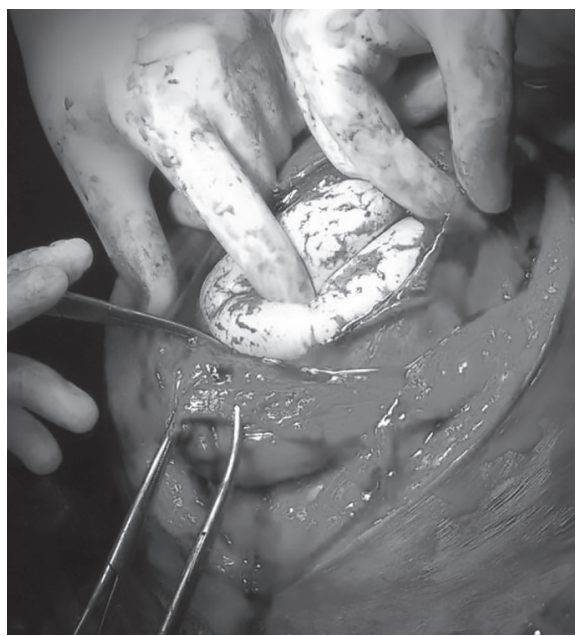

Fig. 2: Uterine incision given with stillbirth baby in transverse lie

\section{DISCUSSION}

Fecolith, fecal impaction in the rectum and colon, causes many complications in pregnancy like dystocia, rupture uterus, and malpresentation. It may also compress the bowel and cause bowel perforation. Prolonged stasis of fecal matter causes impaction and a giant fecolith forms. This obstructs the colon necessitating surgery. A number of questions arise on reading this case, as to the appropriate time when a patient should be delivered, whether to induce labor or prefer spontaneous onset, if a cesarean delivery would have a better outcome, and so on. However, there are no published guidelines for the management of such a case. Hence, we need to review the literature that has been published describing management of such a case.

Rambo, ${ }^{1}$ Etzel et al, ${ }^{2}$ Grasby and Higgins ${ }^{3}$ and Holt and Hendricks ${ }^{4}$ have reported cases of obstructed or dysfunctional labor due to impacted feces where delivery of fetus was achieved after manual disimpaction by removal of feces under anesthesia. Philip Smith for the first time in 1992 reported cesarean delivery for this indication. ${ }^{3}$

In this case, fecal impaction caused obstructed labor, resulting in a hysterotomy in her previous pregnancy and obstructed labor leading to cesarean section for an IUFD in her next pregnancy again, with the cause remaining the same. Obstructed labor and impending uterine rupture warranted a cesarean section in consecutive pregnancy. Intracesarean manual fecal removal was done and bowel was evacuated both the times.

There have been only few published cases on fecal impaction during pregnancy, making this a topic to be studied and further researched into. Based on references of cases published so far, we can deduce:

- The need to anticipate the possibility of dystocia and complications like malpresentations, scar dehiscence, rupture, and fetal compromise

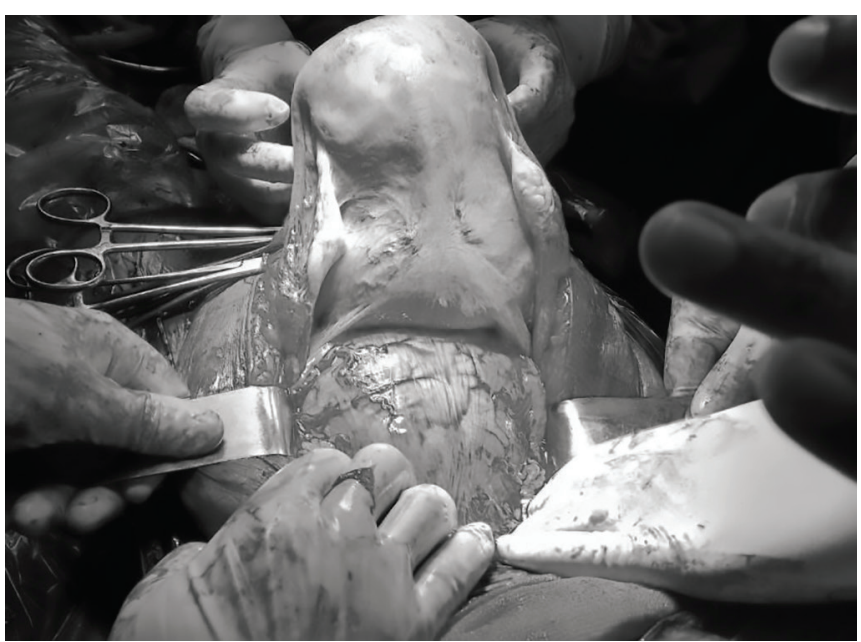

Fig. 3: Dilated sigmoid colon and rectum posteriorly. Uterus and cervix pushed forward

- Manual disimpaction, under continuous fetal monitoring, should be attempted at first, failing which mode of delivery should be reconsidered. $3,5,6$

It has been estimated that approximately 11 to $38 \%$ of pregnant women experience constipation. Pregnancy predisposes women to developing constipation owing to physiologic and anatomic changes in the gastrointestinal tract. Causes of constipation in pregnancy are:

- Rising progesterone levels during pregnancy and reduced motilin hormone levels lead to increase in bowel transit time

- Increased water absorption from the intestines causes stool to dry out

- Decreased maternal activity and iron supplementation

- Later in pregnancy, an enlarging uterus might slow onward movement of feces. ${ }^{7,8}$

For severe constipation in pregnancy leading to megacolon formation, as in our case, requires multidisciplinary approach from surgeons and gynecologist. Conservative measures include a high-fiber, high-water intake; exercise; the use of enemas, bulking agents, and laxatives; and manual disimpaction. If not responded conservatively, then surgical management is required. ${ }^{6}$ In our case, patient went into labor with antepartum hemorrhage with obstruction due to impacted feces, hence cesarean section with manual removal of feces was done.

\section{CONCLUSION}

In this case, the patient underwent consecutive cesarean section for the same indication, which makes constipation during pregnancy a condition for concern. It also compels us to find causes of severe constipation with megacolon, which manifest or are exaggerated during pregnancy.

In cases with obstructed labor with a mass felt per vaginum, large fecoliths should be considered in the 
differential diagnosis. Multidisciplinary team approach is essential for the management of such cases.

\section{REFERENCES}

1. Rambo WW. Dystocia due to fecal impaction resembling a pelvic tumor. Am J Obstet Gynecol 1927 Dec;14(6):812-813.

2. Etzel CA, DiMedio MT, Journey RW. Dystocia caused by fecal impaction. Am J Obstet Gynecol 1954 Aug;68(2): 725-726.

3. Grasby ED, Higgins BK. Megacolon in pregnancy causing dystocia. J Obstet Gynaecol Br Emp 1955 Dec;62(6): 912-913.
4. Holt WA, Hendricks CH. Dysfunctional labor due to fecal impaction. Obstet Gynecol 1969 Oct;34(4):502-505.

5. Grossmann EM, Kaminski DL, Amon E, Longo WE. Idiopathic megarectum complicating pregnancy: report of a case. Am J Gastroenterol 2000 Oct;95(10):2969-2972.

6. Resta P,Nardelli GB, Mincato C, De Bastiani BM. Hirschsprung's disease and pregnancy: a case report. Eur J Obstet Gynecol Reprod Biol 1979 Feb;9(1):51-53.

7. Longo SA, Moore RC, Canzoneri BJ, Robichaux A. Gastrointestinal conditions during pregnancy. Clin Colon Rectal Surg 2010 Jun;23(2):80-89.

8. Cullen G, O'Donoghue D. Constipation and pregnancy. Best Pract Res Clin Gastroenterol 2007;21(5):807-818. 